\title{
Improved Antifouling Properties of Polyamide Nanofiltration Membranes by Reducing the Density of Surface Carboxyl Groups
}

\author{
Yinghui Mo, ${ }^{\dagger}$ Alberto Tiraferri, ${ }^{\ddagger}$ Ngai Yin Yip, ${ }^{\ddagger}$ Atar Adout, ${ }^{\ddagger}$ Xia Huang, ${ }^{* \dagger}{ }^{\dagger}$ and Menachem Elimelech ${ }^{*}, \ddagger$ \\ ${ }^{\dagger}$ State Key Joint Laboratory of Environment Simulation and Pollution Control, School of Environment, Tsinghua University, Beijing \\ 100084, China
}

${ }^{\ddagger}$ Department of Chemical and Environmental Engineering, Yale University, New Haven, Connecticut 06520-8286, United States

Supporting Information

\begin{abstract}
Carboxyls are inherent functional groups of thin-film composite polyamide nanofiltration (NF) membranes, which may play a role in membrane performance and fouling. Their surface presence is attributed to incomplete reaction of acyl chloride monomers during the membrane active layer synthesis by interfacial polymerization. In order to unravel the effect of carboxyl group density on organic fouling, NF membranes were fabricated by reacting piperazine (PIP) with either isophthaloyl chloride (IPC) or the more commonly used trimesoyl chloride (TMC). Fouling experiments were conducted with alginate as a model hydrophilic organic foulant

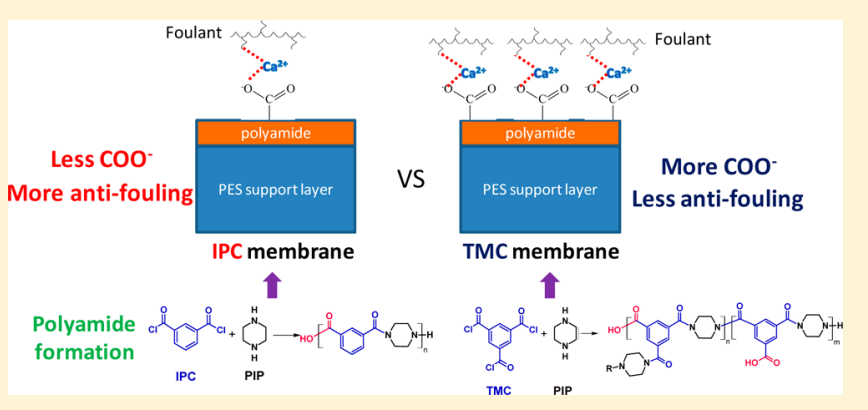
in a solution, simulating the composition of municipal secondary effluent. Improved antifouling properties were observed for the IPC membrane, which exhibited lower flux decline (40\%) and significantly greater fouling reversibility or cleaning efficiency (74\%) than the TMC membrane (51\% flux decline and $40 \%$ cleaning efficiency). Surface characterization revealed that there was a substantial difference in the density of surface carboxyl groups between the IPC and TMC membranes, while other surface properties were comparable. The role of carboxyl groups was elucidated by measurements of foulant-surface intermolecular forces by atomic force microscopy, which showed lower adhesion forces and rupture distances for the IPC membrane compared to TMC membranes in the presence of calcium ions in solution. Our results demonstrated that a decrease in surface carboxyl group density of polyamide membranes fabricated with IPC monomers can prevent calcium bridging with alginate and, thus, improve membrane antifouling properties.
\end{abstract}

\section{INTRODUCTION}

Membrane fouling by effluent organic matter (EfOM) is a major hindrance to the effective application of nanofiltration (NF) technology to the reclamation of municipal secondary effluent. ${ }^{1,2}$ Fouling decreases water productivity, deteriorates permeate quality, and shortens membrane lifespan. ${ }^{3}$ Current operational approaches to cope with membrane fouling include pretreatment of feed solution and/or cleaning of the fouled membranes. ${ }^{3}$ However, these approaches have a significant impact on the cost and operation of the membrane process. ${ }^{4}$ Therefore, it is essential to design membranes that possess optimized surface properties and are less prone to fouling.

Fouling is strongly influenced by the properties of the membrane surface. These characteristics include surface charge, surface roughness, wettability, and surface functional groups specifically interacting with foulants. ${ }^{5}$ In particular, carboxyl groups commonly exist on the surface of thin-film composite (TFC) polyamide NF membranes. ${ }^{5}$ The occurrence of carboxyl groups at the membrane surface is attributed to the membrane fabrication technique. TFC membranes comprise an ultrathin active layer cast on microporous substrate. ${ }^{6,7}$ The ultrathin active layer, which determines the permeability and selectivity of the NF membrane, is fabricated by interfacial polymerization
(IP) of polyamide with amine and acyl chloride monomers as reactants. ${ }^{8,9}$ The unreacted acyl chlorides remaining in the polyamide matrix rapidly hydrolyze into carboxyl groups after the TFC membrane is immersed in water during the fabrication process. $^{10}$

The role that the carboxyl groups play in fouling is conflicting and not well understood. Carboxyl groups contribute to surface negative charge and surface hydrophilicity. It has been reported that negative surface charge and hydrophilicity are desirable membrane properties to reduce fouling. ${ }^{3,11-16}$ On the other hand, in the presence of calcium ions, which are ubiquitous in natural waters and secondary effluents, carboxyl groups can enable the formation of calcium bridges between the membrane surface and organic foulants and, consequently, increase organic fouling. ${ }^{12,17-19}$ Calcium bridging has been demonstrated in numerous studies with a broad range of model organic foulants, such as alginate, ${ }^{20}$ bovine serum albumin (BSA), ${ }^{11}$ humic acid, ${ }^{16}$ and extracellular

Received: September 10, 2012

Revised: November 19, 2012

Accepted: November 20, 2012

Published: December 3, 2012 


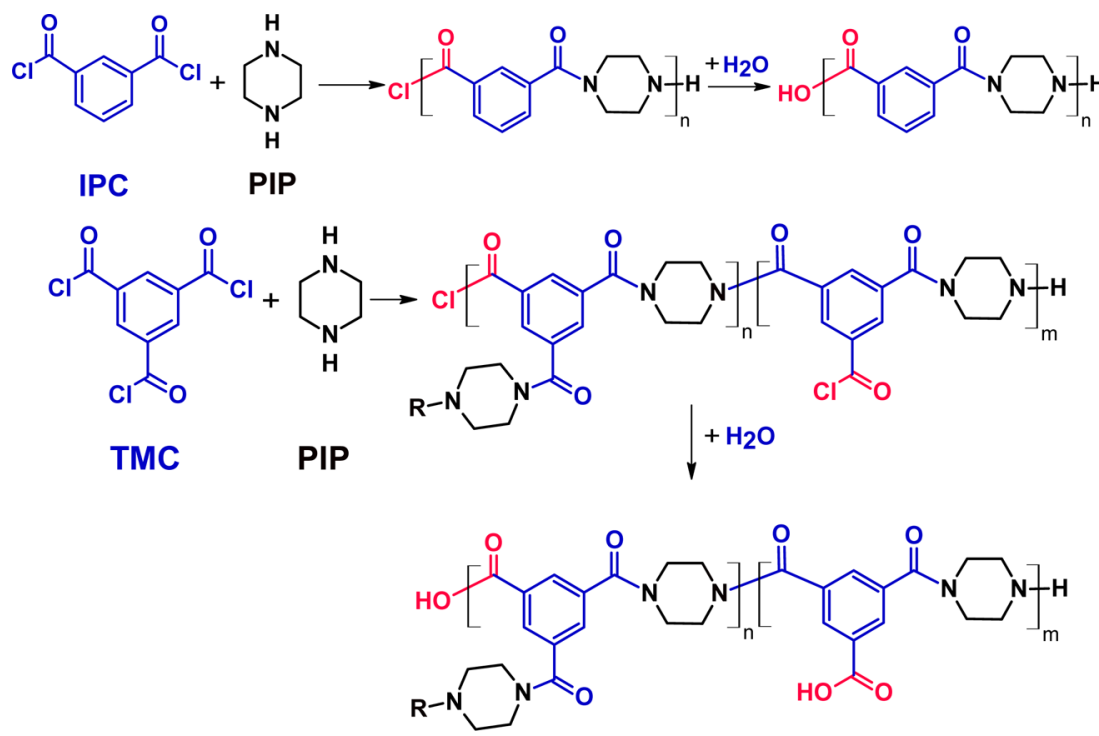

Figure 1. Reaction of trimesoyl chloride (TMC) or isophthaloyl chloride (IPC) with piperazine (PIP) during interfacial polymerization. Acyl chloride moieties react with the amine groups of PIP to form amide linkages. The unreacted acyl chloride moieties are subsequently hydrolyzed into carboxyl groups.

polymeric substances (EPS). ${ }^{21}$ Hence, the impact of membrane surface carboxyl groups on organic fouling is not straightforward and necessitates systematic investigation.

Kasher et al. ${ }^{5}$ have investigated the adsorption of organic foulants on carboxyl groups as well as other common membrane surface functionalities using self-assembled monolayers as model surfaces. The use of self-assembled monolayers provided well-defined and uniform surface chemistry, thereby avoiding physical and chemical surface heterogeneities that are inherent to the interfacial polymerization process. To date, however, there are no published studies on the effect of surface carboxyl groups of TFC membranes on organic fouling.

In order to investigate the influence of membrane surface carboxyl groups on fouling, other surface properties that may contribute to fouling need to be held constant. Therefore, two acyl chloride monomers, trimesoyl chloride (TMC) and isophthaloyl chloride (IPC), were purposely selected for our study. These two monomers have very similar chemical composition and structure, with the only difference being the number of acyl chlorides in each molecule. Thus, we surmise that the density of surface carboxyl groups will be the only property that varies, while other properties remain unchanged.

TMC is the most prevalent monomer employed for interfacial polymerization of polyamide TFC membranes. $^{10,22-26}$ Although less popular, the application of IPC to fabricate TFC membranes can trace its history to the patents published by Cadotte ${ }^{27,28}$ and Sundet ${ }^{29}$ three decades ago. Studies on IPC membranes mainly focused on the membrane separation properties (water permeability and salt rejection), ${ }^{7,30-34}$ surface composition and morphology, ${ }^{30-32}$ or the resistance of the membrane to chlorine attack. ${ }^{35,36}$ The impact of IPC on the density of carboxyl groups on the membrane surface as well as fouling has not yet been explored.

In this study we investigate the potential of using IPC in place of TMC during interfacial polymerization to improve membrane antifouling properties by reducing the carboxyl group density of NF membrane surfaces. NF membranes were fabricated on a commercial ultrafiltration (UF) membrane support with IPC and TMC monomers. Antifouling properties, described by water flux decline and fouling reversibility (indicated by physical cleaning efficiency), of the two membrane types were compared. The membrane surfaces were characterized and the intermolecular forces between the membrane surface and foulants were analyzed to elucidate the role of carboxyl groups in the organic fouling behavior of TFC polyamide NF membranes.

\section{MATERIALS AND METHODS}

Materials and Chemicals. For thin-film composite (TFC) NF membrane fabrication, poly(ether sulfone) (PES) ultrafiltration (UF) membranes (PES20, Sepro Membranes Inc., Oceanside, CA) were used as support layers. The UF membranes were prewet by immersing in deionized (DI) water for approximately $3 \mathrm{~h}$ prior to polyamide fabrication. Piperazine (PIP) and isophthaloyl chloride (IPC) or trimesoyl chloride (TMC) were used as monomers for interfacial polymerization of polyamide (Sigma-Aldrich, St. Louis, MO). Hexane was used as solvent to dissolve IPC or TMC (Sigma-Aldrich). For membrane testing, sodium chloride $(\mathrm{NaCl})$, calcium chloride $\left(\mathrm{CaCl}_{2}\right)$, and sodium bicarbonate $\left(\mathrm{NaHCO}_{3}\right)$ were purchased from J.T. Baker (Phillipsburg, NJ). Magnesium sulfate $\left(\mathrm{MgSO}_{4}\right)$, sodium alginate, and toluidine blue $\mathrm{O}(\mathrm{TBO})$ were purchased from Sigma-Aldrich. The inorganic salts were of reagent grade and, when not stated otherwise, all solutions were prepared by dissolution in DI water from a Milli-Q ultrapure water purification system (Millipore, Billerica, MA).

Thin-Film Composite Nanofiltration Membrane Fabrication. TFC NF membranes were fabricated by forming a polyamide active layer on top of the commercial PES support membrane via interfacial polymerization. The chemical structures of the monomers used during interfacial polymerization (PIP, IPC, and TMC) are presented in Figure 1. The reaction between amine functional groups in PIP and acyl chloride moieties in IPC or TMC produces amide linkages, thus forming polyamide. ${ }^{37}$ The unreacted acyl chloride groups are hydrolyzed into carboxyl groups after contact with water. ${ }^{10,37,38}$ 
To fabricate the active layers, the PES support membrane was tightly taped onto a clean glass plate with laboratory tape, with the skin layer facing upward. Approximately $10 \mathrm{~mL}$ of $1 \mathrm{wt}$ $\%$ PIP solution was dispensed onto the surface of the support layer and was allowed to contact for $90 \mathrm{~s}$. Then the PIP solution was drained off by positioning the glass plate vertically. An air knife was used to remove the excess PIP solution from the surface of the support layer. Next, the PIP-saturated support layer was immersed in $0.1 \mathrm{wt} \%$ IPC or $0.13 \mathrm{wt} \%$ TMC for 30 s. Finally, the newly formed membranes were air-dried for 120 s. The same molar concentrations $(3.2 \mathrm{mM})$ were used for both TMC and IPC. The resultant TFC NF membranes were rinsed thoroughly with DI water to remove chemical residues and then stored in DI water at $4{ }^{\circ} \mathrm{C}$ for at least $24 \mathrm{~h}$ prior to use. The interfacial polymerization conditions are tabulated in Supporting Information. Similar protocols for TFC membrane fabrication were described in our previous publications. ${ }^{39,40}$

Membrane Characterization. Recently, we have demonstrated the use of TBO, a cationic dye $\left(\mathrm{C}_{15} \mathrm{H}_{16} \mathrm{~N}_{3} \mathrm{~S}^{+}\right.$, molecular mass $270 \mathrm{~g} / \mathrm{mol}$ ), to determine the concentration of negatively charged functional groups on membrane surfaces. ${ }^{41}$ The surface carboxyl group density of the fabricated membranes was measured via the TBO technique. TFC NF membranes were taped onto a clean glass plate with the active layer facing upward. A TBO solution ( $2 \mathrm{mM})$ was freshly prepared by dissolving $\mathrm{TBO}$ in aqueous sodium hydroxide $(\mathrm{NaOH})$ at $\mathrm{pH}$ 11. The TFC NF membranes were contacted with the TBO solution (approximately $1 \mathrm{~mL}$ of TBO solution for $1 \mathrm{~cm}^{2}$ of membrane area) for $3 \mathrm{~min}$ at room temperature $\left(23^{\circ} \mathrm{C}\right)$. Then, the membranes were detached from the glass plate, rinsed, and immersed in a dye-free $\mathrm{pH} 11(\mathrm{NaOH})$ solution $(>4 \mathrm{~h})$ to wash off the unbound TBO molecules. Next, two 1-in. diameter samples were punched for each surface and placed into $10 \mathrm{~mL}$ of $0.2 \mathrm{M} \mathrm{NaCl}$ solutions at $\mathrm{pH} 2$ (adjusted with hydrochloric acid) for $30 \mathrm{~min}$ while stirring. This step releases the TBO bound to the carboxyl groups into the solution. The resultant solutions were analyzed by optical density at a $630 \mathrm{~nm}$ wavelength. Light absorption was converted to TBO concentration by use of a calibration curve, and the membrane surface carboxyl group density was calculated by dividing the number of TBO molecules by the membrane planar area. ${ }^{41}$

Zeta potential of membrane surfaces was determined on a streaming potential analyzer (BI-EKA, Brookhaven Instruments Co., Holtsville, NY). ${ }^{42,43}$ The membrane surface was equilibrated with the testing solution for at least $45 \mathrm{~min}$ before measurements. Two solutions were tested: (i) a calcium-free solution containing $9 \mathrm{mM} \mathrm{NaCl}$ and $1 \mathrm{mM} \mathrm{NaHCO}_{3}$ and (ii) a solution comprising $6 \mathrm{mM} \mathrm{NaCl}, 1 \mathrm{mM} \mathrm{NaHCO}$, and $1 \mathrm{mM}$ $\mathrm{CaCl}_{2}$. Measurements were conducted for three separately cast membranes.

A goniometer (VCA video contact angle system, Billerica, $\mathrm{MA}$ ) was used to measure the contact angle between the fabricated TFC NF membrane surface and a droplet of DI water. Membranes were air-dried overnight at $45{ }^{\circ} \mathrm{C}$ before measurement. Measurements were conducted at seven different spots of the membrane surface and the results were averaged.

A multimode atomic force microscope (AFM, Veeco Metrology Group, Santa Barbara, CA) was used to determine the surface roughness of the fabricated TFC NF membranes in tapping mode. The silicon probe (Tap300AI-G, Budget Sensors, Sofia, Bulgaria) had a spring constant of $40 \mathrm{~N} / \mathrm{m}$, resonance frequency of $300 \mathrm{~Hz}$, and cantilever length of 125 $\mu \mathrm{m}$. Membranes were air-dried overnight before scanning.
Imaging was performed on a scan area of $5 \mu \mathrm{m} \times 5 \mu \mathrm{m}$ with a tip velocity of $5.82 \mu \mathrm{m} / \mathrm{s}$ and scan rate of $0.5 \mathrm{~Hz}$. Membrane roughness was averaged from five randomly selected spots and is expressed as the root-mean-square roughness $\left(R_{\mathrm{rms}}\right)$, average roughness $\left(R_{\mathrm{a}}\right)$, maximum roughness $\left(R_{\max }\right)$, and surface area difference (SAD, the calculated difference between actual surface area and projected area).

The water permeability and salt rejection of the TFC NF membranes were measured on a bench-scale cross-flow system described in our previous studies. ${ }^{11}$ The membrane coupons were loaded into a rectangular plate-and-frame cell with an active area of $20.02 \mathrm{~cm}^{2}$. The temperature of the feed solution was maintained at $25 \pm 0.5{ }^{\circ} \mathrm{C}$ by a recirculating chiller. Before measurement, the membranes were compacted with DI water for at least $14 \mathrm{~h}$ until the flux was stable. The applied hydraulic pressure was 20.7 bar ( $300 \mathrm{psi}$ ) for the IPC membranes and 6.9 bar (100 psi) for the TMC membranes, due to the different intrinsic water permeabilities of the two membrane types. The water permeability was calculated by dividing the measured water flux after compaction by the applied hydraulic pressure. Solutions of $25 \mathrm{mM} \mathrm{NaCl}$ or $25 \mathrm{mM} \mathrm{MgSO}_{4}$ were used to determine the selectivity of membranes at a cross-flow velocity of $21.4 \mathrm{~cm} / \mathrm{s}$. Salt rejection was calculated from the measured conductivities of the feed and permeate solutions. The measured conductivities were converted to salt concentrations by use of a calibration curve.

Membrane Fouling Protocol. Fouling experiments were performed in the same cross-flow system described above. Before fouling, membranes were equilibrated with a background electrolyte solution, which contained $1 \mathrm{mM} \mathrm{CaCl}_{2}, 16$ $\mathrm{mM} \mathrm{NaCl}$, and $1 \mathrm{mM} \mathrm{NaHCO}$ (total ionic strength $20 \mathrm{mM}$, $\mathrm{pH} 8.0 \pm 1$ ). During this process, the cross-flow velocity was maintained at $12.8 \mathrm{~cm} / \mathrm{s}$ while the water flux was adjusted to 45 $\mathrm{L} \cdot \mathrm{m}^{-2} \cdot \mathrm{h}^{-1}$ by varying the applied hydraulic pressure. To reach the target water flux, the IPC membranes were operated at approximately $15.2-17.3$ bar (220-250 psi), while the TMC membranes were operated in the range of 6.9-9.0 bar (100$130 \mathrm{psi}$ ). After a 4-h equilibration, $100 \mathrm{~mL}$ of alginate stock solution $(1 \mathrm{~g} / \mathrm{L})$ was added into the background electrolyte solution to achieve an alginate concentration of $20 \mathrm{mg} / \mathrm{L}$ and to initiate fouling. The fouling stage was continued for $18 \mathrm{~h}$, during which the flux was recorded at fixed intervals.

Evaluation of Fouling Reversibility. Fouling reversibility was evaluated through physical cleaning experiments. At the end of the fouling run, the feed solution was replaced by a cleaning solution with the same ionic compositions as the background electrolyte solution used in the equilibration step (i.e., no alginate). Physical cleaning was performed without applied hydraulic pressure and at an increased cross-flow velocity of $51.2 \mathrm{~cm} / \mathrm{s}$ for $30 \mathrm{~min}$. Subsequently, the cleaning solution was drained, and fresh background electrolyte solution was filtered through the membrane to determine the permeate flux of the cleaned membrane. Cleaning efficiency, $\eta$, was calculated from

$$
\eta(\%)=\frac{J_{c}-J_{a}}{J_{b}-J_{a}} \times 100
$$

where $J_{\mathrm{b}}$ is the water flux with background electrolyte solution before fouling, $J_{\mathrm{a}}$ is the water flux with background electrolyte solution after fouling, and $J_{\mathrm{c}}$ is the water flux after cleaning. Cleaning efficiency was used as an indicator for fouling reversibility. ${ }^{44}$ 
Interaction Force Measurement by Atomic Force Microscope. The interaction forces between the organic foulant and membrane surface were determined by a Nanoscope III multimode atomic force microscope (AFM, Digital Instruments, Santa Barbara, CA). A carboxylatemodified latex (CML) particle (Interfacial Dynamics Corp., Portland, OR), rich in surface carboxyl groups, was used as a surrogate of alginate. ${ }^{45}$ The AFM probe was prepared by gluing the CML particle (radius of $2 \mu \mathrm{m}$ ) to the end of a commercial $\mathrm{SiN}$ tipless cantilever with a spring constant of $0.06 \mathrm{~N} / \mathrm{m}$ (Veeco Metrology Group, Santa Barbara, CA). A fluid cell was used to allow force measurements in liquid at the desired solution chemistry. The two solutions consisted of $19 \mathrm{mM}$ $\mathrm{NaCl}$ and $1 \mathrm{mM} \mathrm{NaHCO}_{3}$ or of $16 \mathrm{mM} \mathrm{NaCl}, 1 \mathrm{mM}$ $\mathrm{NaHCO}_{3}$, and $1 \mathrm{mM} \mathrm{CaCl}$ (i.e., same ionic strength but without and with calcium ions, respectively). Force measurements were performed at five randomly selected spots on each membrane surface, with 25 measurements for each spot. Details about interaction force measurements by AFM can be found in our previous studies. ${ }^{45-47}$

\section{RESULTS AND DISCUSSION}

Permeability and Selectivity of Fabricated Membranes. The average water permeability of the TMC membranes was $6.2 \mathrm{~L} \cdot \mathrm{m}^{-2} \cdot \mathrm{h}^{-1} \cdot \mathrm{bar}^{-1}$, comparable to reported values of TFC NF membranes fabricated from the same monomers (PIP and TMC). ${ }^{8,16,22-26,38}$ Compared to the TMC membrane, the IPC membrane had a lower average water permeability of $2.9 \mathrm{~L} \cdot \mathrm{m}^{-2} \cdot \mathrm{h}^{-1} \cdot \mathrm{bar}^{-1}$. Lower permeability of IPC membranes has also been reported by Cadotte ${ }^{28}$ and Saha and Joshi, ${ }^{8}$ where it was suggested that a mixture of IPC and TMC for TFC membrane fabrication can improve water permeability. Table 1 presents the complete data for water permeability and solute rejection of the fabricated TFC NF membranes.

The average $\mathrm{NaCl}$ rejection, with a $25 \mathrm{mM} \mathrm{NaCl}$ feed solution, was $30.2 \%$ at 6.9 bar (100 psi) for TMC membranes and $51.8 \%$ at 20.7 bar (300 psi) for IPC membranes. However, the rejection trend was reversed for $\mathrm{MgSO}_{4}$ salt, with the TMC and IPC membranes exhibiting average rejections of $94.5 \%$ and $77.7 \%$, respectively, under the same applied hydraulic pressures as the $\mathrm{NaCl}$ rejection tests. The salt separation mechanisms of NF membranes include steric (size) exclusion and electrostatic partitioning effects (Donnan equilibrium and dielectric exclusion). ${ }^{48-51}$ For our negatively charged NF membranes, electrostatic repulsion plays a more important role in the rejection of salts containing multivalent anions than salts with monovalent anions. ${ }^{52}$ The higher rejection of $\mathrm{NaCl}$ by the IPC membrane is attributed to its denser active layer structure, resulting in enhanced size exclusion. However, as discussed later, the TMC membrane is more negatively charged than the IPC membrane, giving rise to stronger electrostatic repulsion of the sulfate anions, which results in a higher rejection of $\mathrm{MgSO}_{4}{ }^{48}$

Fouling Behavior and Reversibility. Fouling behavior of the TMC and IPC membranes with alginate as a model organic foulant is described in Figure 2. An initial flux of $45 \mathrm{~L} \cdot \mathrm{m}^{-2} \cdot \mathrm{h}^{-1}$ was used for the fouling experiments with both membranes by adjusting the applied hydraulic pressure. The IPC membranes exhibited a slower flux decline than the TMC membranes, and the water flux at the end of the fouling step (after $18 \mathrm{~h}$ ) was also higher for the IPC membranes $\left(25-30 \mathrm{~L} \cdot \mathrm{m}^{-2} \cdot \mathrm{h}^{-1}\right.$ for IPC compared to $20 \mathrm{~L} \cdot \mathrm{m}^{-2} \cdot \mathrm{h}^{-1}$ for TMC). The flux decline data
Table 1. Water Permeability, Salt Rejection, and Surface Characteristics of Fabricated TMC and IPC Nanofiltration Membranes

\begin{tabular}{|c|c|c|}
\hline & $\mathrm{TMC}^{a}$ & $\mathrm{IPC}^{b}$ \\
\hline water permeability ${ }^{c}\left(\mathrm{~L} \cdot \mathrm{m}^{-2} \cdot \mathrm{h}^{-1} \cdot \mathrm{bar}^{-1}\right)$ & $6.2 \pm 0.8$ & $2.9 \pm 0.4$ \\
\hline$R(\mathrm{NaCl})^{d}(\%)$ & $30.2 \pm 2.8$ & $51.8 \pm 4.3$ \\
\hline$R\left(\mathrm{MgSO}_{4}\right)^{d}(\%)$ & $94.5 \pm 1.4$ & $77.7 \pm 3.4$ \\
\hline contact angle $^{e}(\mathrm{deg})$ & $66.0 \pm 10.8$ & $66.2 \pm 3.7$ \\
\hline$\zeta$ potential $^{f}(\mathrm{mV})$ & $-0.4 \pm 1.8$ & $-1.0 \pm 1.9$ \\
\hline \multicolumn{3}{|c|}{ Surface Roughness } \\
\hline$R_{\mathrm{rms}}(\mathrm{nm})$ & $48.6 \pm 11.2$ & $59.4 \pm 12.8$ \\
\hline$R_{\mathrm{a}}(\mathrm{nm})$ & $37.4 \pm 8.2$ & $45.2 \pm 10.4$ \\
\hline$R_{\max }(\mathrm{nm})$ & $349.7 \pm 65.7$ & $394.3 \pm 84.7$ \\
\hline $\mathrm{SAD}^{g}(\%)$ & $22.8 \pm 2.6$ & $14.8 \pm 5.3$ \\
\hline
\end{tabular}

${ }^{a}$ Average \pm standard deviation of up to 10 membrane samples. ${ }^{b}$ Average \pm standard deviation of up to six membrane samples. ${ }^{c}$ Water permeabilities were determined with DI water at $25{ }^{\circ} \mathrm{C}$. Applied pressures during measurements of water permeabilities were 6.9 bar (100 psi) for TMC membrane and 20.7 bar $(300 \mathrm{psi})$ for IPC membrane. ${ }^{d}$ Determined by conductivity measurements in cross-flow filtration with $25 \mathrm{mM} \mathrm{NaCl}$ or $\mathrm{MgSO}_{4}$ feed solution at $25^{\circ} \mathrm{C}$. Applied pressure during measurements of salt rejection was the same as that used for determining the water permeabilities. ${ }^{e}$ Contact angle was measured with deionized water. ${ }^{f_{\text {Solution }}}$ chemistry for the $\zeta$ potential measurements: $1 \mathrm{mM} \mathrm{CaCl}_{2}, 6 \mathrm{mM} \mathrm{NaCl}, 1 \mathrm{mM} \mathrm{NaHCO}_{3}$ (i.e., total ionic strength of $10 \mathrm{mM}$ ); $\mathrm{pH} 8.0 \pm 0.1$. Due to instrument limitation, an ionic strength of $10 \mathrm{mM}$ is the maximum value that can be employed. ${ }^{g}$ Surface area difference, the calculated difference between actual surface area and projected area

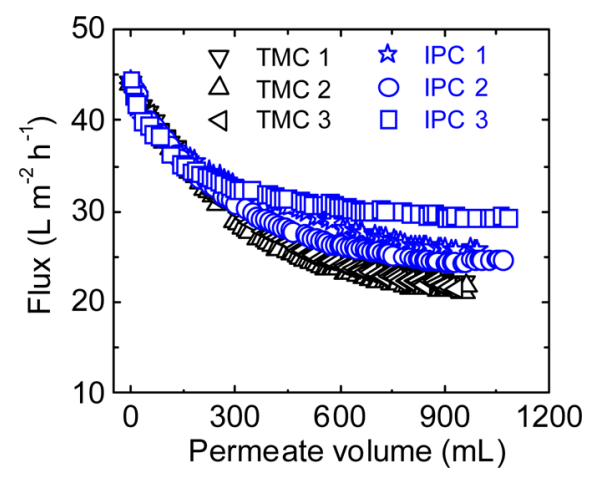

Figure 2. Flux decline behavior of TMC and IPC membranes during fouling tests. The following feed solution was used for fouling runs: alginate, $20 \mathrm{mg} / \mathrm{L} ; \mathrm{CaCl}_{2}, 1 \mathrm{mM} ; \mathrm{NaCl}, 16 \mathrm{mM} ; \mathrm{NaHCO}_{3}, 1 \mathrm{mM} ; \mathrm{pH}$, $8.0 \pm 0.1$. Hydraulic pressure was adjusted to achieve an initial permeate flux of $45 \mathrm{~L} \cdot \mathrm{m}^{-2} \cdot \mathrm{h}^{-1}$. Other experimental conditions: crossflow velocity, $12.8 \pm 0.5 \mathrm{~cm} / \mathrm{s}$; temperature, $25 \pm 0.5^{\circ} \mathrm{C}$. Each fouling run was carried out for $18 \mathrm{~h}$.

suggest that the IPC membranes may be more resistant to fouling than the TMC membranes.

The accelerated fouling experiments were performed at high initial permeate flux, resulting in severe fouling of both of the IPC and TMC membranes. Under these conditions, with a significant permeation drag imposed on the foulants, accumulation of foulants at the membrane surface and subsequent flux decline are inevitable. However, flux decline behavior is not the appropriate measure for assessing the antifouling property of membranes, but rather flux recovery behavior, which is indicative of fouling reversibility. ${ }^{53} \mathrm{We}$ evaluated the reversibility of fouling by carrying out physical cleaning experiments of the fouled membranes without water 
permeation (i.e., with no hydraulic pressure). Physical cleaning of the membranes was accomplished by increasing the crossflow velocity of the same feed solution but without alginate, thereby enhancing hydrodynamic shear at the feed-membrane interface to remove foulants. The reversibility of fouling was evaluated by determining the flux recovery after cleaning (eq 1).

The average cleaning efficiency of the IPC membranes was around $74 \%$, much higher than that of the TMC membranes (40\%) (Figure 3). Note that cleaning experiments were

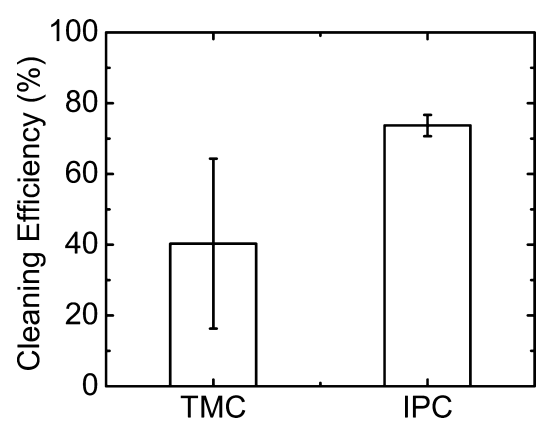

Figure 3. Comparison of cleaning efficiency (calculated from eq 1) of fouled TMC and IPC membranes. The feed solution used for physical (hydraulic) cleaning has identical ionic composition as the fouling solution described in Figure 2 but without the alginate foulant. Other experimental conditions: hydraulic pressure, $\approx 0$ bar $(<0.7 \mathrm{bar})$; crossflow velocity, $51.2 \pm 6.4 \mathrm{~cm} / \mathrm{s}$; temperature, $20 \pm 1{ }^{\circ} \mathrm{C}$; cleaning time, $30 \mathrm{~min}$. The cleaning efficiency of TMC and IPC membranes was averaged from five and three samples, respectively.

repeated three times for the IPC membranes and five times for the TMC membranes. Two additional runs were conducted for the TMC membranes because these membranes exhibited greater variability in cleaning. The cleaning efficiencies for the five runs were $76 \%, 23 \%, 16 \%, 49 \%$ and $37 \%$, yielding a wide distribution (larger error bar in Figure 3). Statistical analysis $(t$ test) has indicated that the cleaning efficiency for the IPC membrane was significantly higher than that for the TMC membrane ( $P$-value of 0.035$)$. Therefore, despite the variability in cleaning efficiency of the TMC membrane, we conclude that the IPC membrane exhibited greater fouling reversibility and hence more antifouling properties.

What Causes the Difference in Antifouling Properties? Fouling and cleaning are governed by the physical and chemical interaction forces that foulants experience. Since alginate macromolecules are relatively large (molecular mass of $12-80 \mathrm{kDa}){ }^{54}$ they will be completely rejected by the fabricated NF membranes. Consequently, membrane fouling will take place only at the membrane surface. The observed difference in antifouling properties of the IPC and TMC membranes is directly related to the membrane surface-foulant interactions. In general, membrane surface-foulant interactions may include electrostatic and van der Waals forces, hydrophobic interaction, and bridging between carboxyl groups via calcium ions. ${ }^{55}$ The extent and importance of the various types of interaction forces that a given foulant experiences (alginate in this study) are greatly affected by the membrane surface properties. We characterized the membrane $\zeta$ potential, wettability, carboxyl group density, and surface roughness to better understand the difference in fouling behavior of our IPC and TMC NF membranes. a. Contact Angle, $\zeta$ Potential, and Surface Roughness Do Not Explain Antifouling Properties. The contact angles of deionized water on the surfaces of IPC and TMC membranes were almost identical $\left(66^{\circ}\right.$, Table 1$)$ and comparable to values reported in literature. ${ }^{8}$ Both IPC and TMC molecules comprise benzene rings with acyl chloride groups, and the only difference between the two molecules is the number of acyl chloride groups (Figure 1). Therefore, the polyamide layers of TMC and IPC membranes have similar chemical composition and wettability. As fouling and cleaning experiments were performed with a feed solution containing calcium ions, $\zeta$ potentials were also determined in the presence of calcium $(6$ $\mathrm{mM} \mathrm{NaCl}, 1 \mathrm{mM} \mathrm{CaCl}_{2}$, and $1 \mathrm{mM} \mathrm{NaHCO}_{3}, \mathrm{pH} 8.0 \pm 0.1$ ). Both IPC and TMC membrane surfaces had nearly neutral charge due to the adsorption of calcium ions, suggesting that electrostatic interactions of the membrane with foulants were negligible during fouling. The surface roughness of the IPC membrane, indicated by the values of $R_{\mathrm{rms}}, R_{\mathrm{a}}$, and $R_{\max }$, was slightly higher than that of the TMC membrane (Table 1). A larger value of surface roughness is often correlated with more fouling; ${ }^{56}$ however, an opposite trend was observed in this study. On the basis of the above observations, we conclude that contact angle, $\zeta$ potential, and surface roughness cannot explain the difference in fouling behavior between IPC and TMC membranes.

b. Lower Membrane Surface Carboxyl Group Density Increases Antifouling Properties. The density of carboxyl groups at the membrane surface was analyzed by two different techniques. In the first method, we quantified the carboxyl group density by measuring the number of bound cationic TBO molecules with carboxyl groups, with the assumption that the ratio of bound TBO molecules to carboxyl groups was $1: 1{ }^{41}$ The bulky size of TBO and the shorter contact time hindered the diffusion of TBO into the polyamide active layer, such that TBO solely interacted with the surface carboxyl groups that were available for interaction with the foulants. ${ }^{41}$ In the second method, we determined the $\zeta$ potential of the membrane surface, which allows qualitative comparison of the negatively charged groups on the IPC and TMC membranes. Two solutions having a total ionic strength of $10 \mathrm{mM}$ but different ionic compositions were employed for the $\zeta$ potential evaluation: (i) $9 \mathrm{mM} \mathrm{NaCl}$ and $1 \mathrm{mM} \mathrm{NaHCO}_{3}$ and (ii) $6 \mathrm{mM}$ $\mathrm{NaCl}, 1 \mathrm{mM} \mathrm{NaHCO}$, and $1 \mathrm{CaCl}_{2}$.

The TBO method results show that the carboxyl group density of the IPC membrane $\left(5.7 \pm 2.4 \mathrm{~nm}^{-2}\right)$ was approximately half that of the TMC membrane $(11.2 \pm 1$ $\mathrm{nm}^{-2}$ ) (Figure 4A). Furthermore, the presence of calcium ions caused a decrease in the absolute value of $\zeta$ potential of the IPC membrane by $1.7 \pm 0.93 \mathrm{mV}$, much smaller than the decrease observed for the TMC membrane (by $6.8 \pm 2.0 \mathrm{mV}$ ) (Figure 4B). Calcium ions reduced the $\zeta$ potential by specific adsorption to the membrane carboxyl groups. Therefore, the pronounced reduction in $\zeta$ potential by calcium ions for the TMC membranes implies higher membrane carboxyl group density, which is in agreement with the direct charge quantification by the TBO method (Figure 4A).

Carboxyl groups in the polyamide active layer originate from the unreacted acyl chloride groups during membrane fabrication as shown in Figure 1. Once the polyamide layer is brought into contact with water, the unreacted acyl chloride groups remaining in the polyamide structure hydrolyze to form carboxyl groups. For the IPC membranes, carboxyl groups are mostly present at the end point of the polyamide chain. Due to 

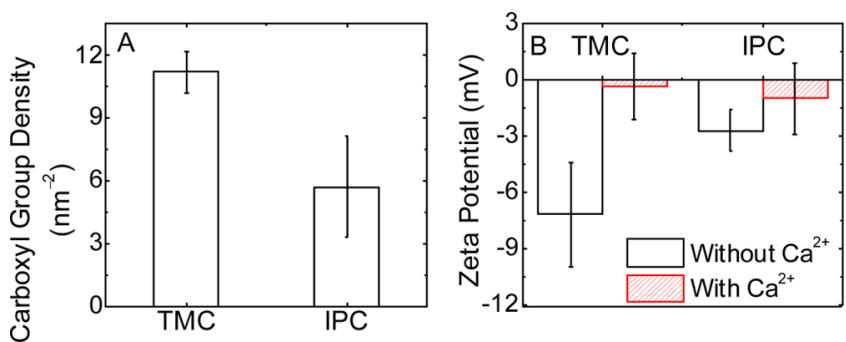

Figure 4. (A) Carboxyl group density and (B) effect of $\mathrm{Ca}^{2+}$ on $\zeta$ potential of TMC and IPC membranes. (A) For carboxyl group density measurements by the TBO method, the solution for the binding step had $2 \mathrm{mM}$ TBO and $\mathrm{pH} 11$ (adjusted by $\mathrm{NaOH}$ ). Membranes were allowed to contact the TBO solution for $3 \mathrm{~min}$ and then exposed to $0.2 \mathrm{M} \mathrm{NaCl}$ concentration at $\mathrm{pH} 2$ (adjusted by $\mathrm{HCl}$ ) foe the release step. (B) For $\zeta$ potential measurements, the solution chemistries used were kept at a total ionic strength of $10 \mathrm{mM}$ and $\mathrm{pH}$ $8.0 \pm 0.1$ as follows: in the absence of calcium ions, $9 \mathrm{mM} \mathrm{NaCl}$ and 1 $\mathrm{mM} \mathrm{NaHCO}$; in the presence of calcium ions, $1 \mathrm{mM} \mathrm{CaCl}_{2}, 6 \mathrm{mM}$ $\mathrm{NaCl}$, and $1 \mathrm{mM} \mathrm{NaHCO}$.

the presence of a third acyl chloride in TMC, carboxyl groups in the TMC membranes can also be found in the middle section of a polyamide chain when cross-linking of the polyamide is incomplete. Hence, the TMC membranes have a greater surface density of carboxyl groups than the IPC membranes, in agreement with the observations in Figure 4.

Our fouling experiments were performed with feed solutions containing calcium ions to simulate the ionic composition of municipal secondary effluent. Calcium ions bind effectively with carboxyl groups through complex formation. ${ }^{12}$ Specifically, calcium ions form bridges between carboxyl groups of alginate molecules and those of the polyamide surface, as well as among alginate molecules, thus resulting in formation of a compact alginate fouling layer on the membrane surface and hence significant fouling. ${ }^{20,45}$ Due to its lower surface carboxyl group density, less calcium bridges form between alginate and the IPC membrane surface, resulting in a more reversible fouling layer that can be easily detached by simple hydraulic cleaning.

To summarize, because fouling of the NF membranes originates from the accumulation of alginate on the membrane, the antifouling properties of the IPC and TMC membranes are dominated by their surface characteristics. The IPC and TMC membranes exhibited no differences in their contact angle, $\zeta$ potential, and surface roughness, indicating that the better antifouling resistance of the IPC membrane is attributed to the lower surface carboxyl group density.

Direct Force Measurements Confirm the Role of Membrane Carboxyl Group Density. Adhesion force measurements by AFM are presented to support our proposed antifouling mechanism of the IPC membrane. A carboxylate-
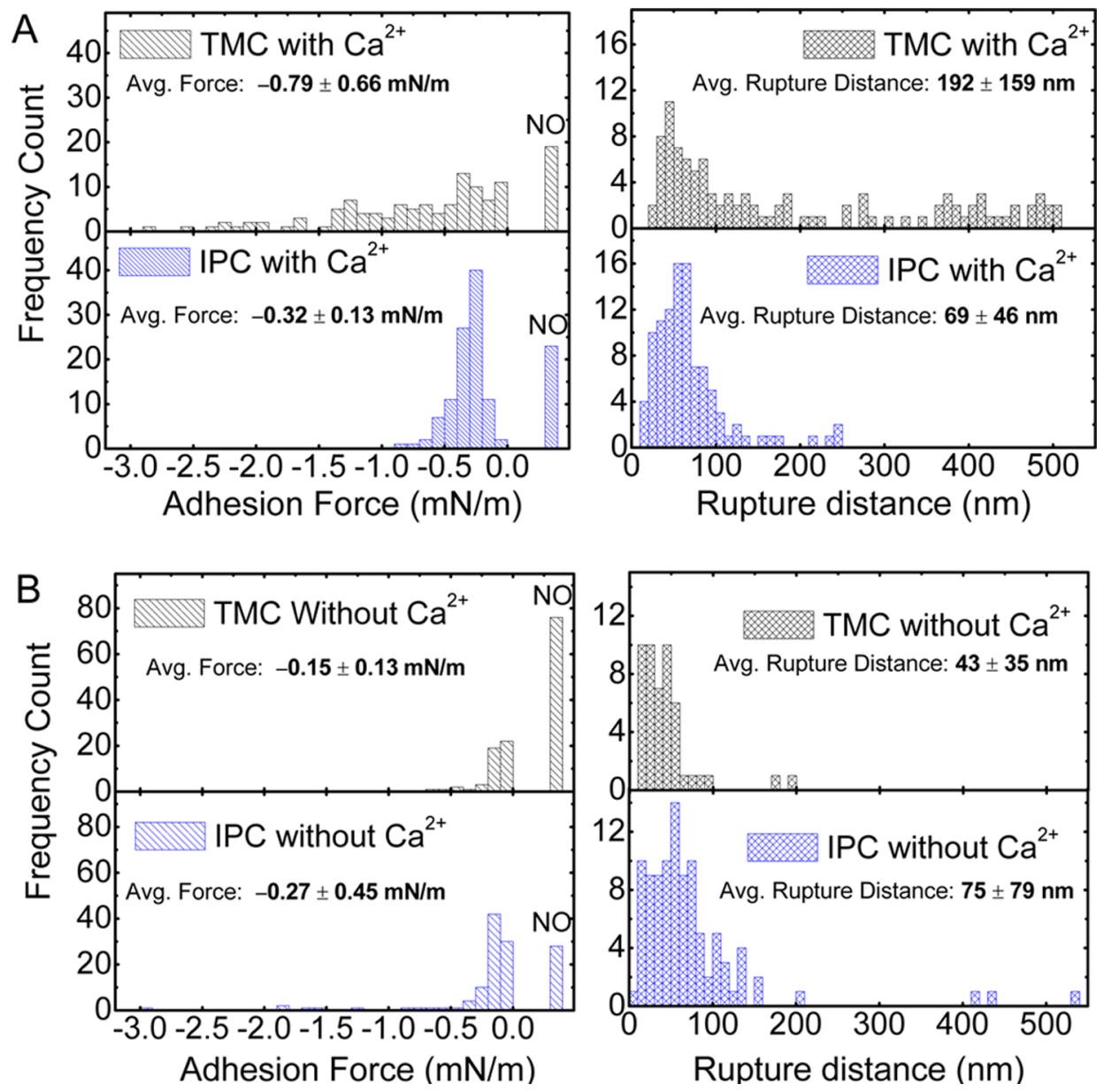

Figure 5. Distributions of adhesion forces and rupture distance in solutions in the (A) presence and (B) absence of calcium ions. The solution chemistries used for the AFM measurements were kept at a total ionic strength of $20 \mathrm{mM}$ and $\mathrm{pH} 8.0 \pm 0.1$ as follows: in the presence of calcium ions, $1 \mathrm{mM} \mathrm{CaCl}_{2}, 16 \mathrm{mM} \mathrm{NaCl}$, and $1 \mathrm{mM} \mathrm{NaHCO}_{3}$; in the absence of calcium ions, $19 \mathrm{mM} \mathrm{NaCl}$ and $1 \mathrm{mM} \mathrm{NaHCO}_{3}$. 
modified latex (CML) AFM particle probe was used as a surrogate for the alginate foulant as described in our previous publication. ${ }^{45}$ From analysis of the force versus distance curve during retraction of the particle probe, we present two important parameters (Figure 5): (i) the adhesion force, which represents the maximum attractive force between the probe and the membrane surface, and (ii) the rupture distance, which represents the maximum extension distance where the probe-surface interaction becomes zero.

In the presence of calcium ions, the adhesion forces between the AFM foulant probe and the TMC membrane surface had a significantly wider distribution than those for the IPC membrane (Figure 5A). Specifically, adhesive forces as high as $2.9 \mathrm{mN} / \mathrm{m}$ were measured for the TMC membrane, whereas the most adhesive force for the IPC membrane was $0.86 \mathrm{mN} /$ $\mathrm{m}$. Also, the average adhesion force for the IPC membrane was $0.32 \mathrm{mN} / \mathrm{m}$, significantly lower than that for the TMC membrane $(0.79 \mathrm{mN} / \mathrm{m})$. A similar trend was observed for the rupture distance, with the TMC membrane exhibiting a much wider distribution of rupture distances and a much higher average rupture distance $(192 \mathrm{~nm})$ than the IPC membrane (69 $\mathrm{nm})$.

In the absence of calcium ions but at the same total ionic strength, the adhesion force and rupture distance for the TMC membrane decreased remarkably from 0.79 to $0.15 \mathrm{mN} / \mathrm{m}$ and from 192 to $43 \mathrm{~nm}$, respectively (Figure 5B). This observation confirms the dominant role of calcium ions in fouling of the TMC membranes. On the contrary, the distribution of forces and rupture distances hardly changed for the IPC membrane in the absence of calcium ions. Moreover, the average adhesion force and rupture distance for the IPC membrane were not statistically different with and without calcium: 0.32 versus 0.27 $\mathrm{mN} / \mathrm{m}$ for the average adhesion force and 69 versus $75 \mathrm{~nm}$ for the rupture distance.

Our previous studies demonstrated the paramount role of calcium bridges, with measurement of strong adhesion forces and long rupture distances for membranes exposed to solutions with alginate or humic acid and calcium ions. ${ }^{45,47}$ Calcium bridges can form only when the distance between the AFM probe and the membrane surface is sufficiently small. However, once formed, adhesion forces can be observed over a long distance when the AFM probe is retracted from the membrane surface (i.e., long rupture distances in Figure 5A). The results presented in Figure 5 provide compelling evidence for the formation of calcium bridges with the TMC membranes but not with the IPC membrane. The AFM results are in striking agreement with both the antifouling properties and the surface carboxyl group densities of the two TFC membranes.

Implications. Due to superior performance compared to integrally skinned asymmetric membranes, polyamide TFC NF membranes have captured the majority of the market share. For wastewater reclamation application, the overarching objective is to design membranes with robust antifouling properties as well as high water permeability and selectivity. The enhanced antifouling of the IPC membranes was achieved at a cost of reduced water permeability. Therefore, the interfacial polymerization protocol needs to be purposefully adjusted to optimize the water permeability. This can be achieved by using a combination of TMC, IPC, and/or other monomers. In addition to utilizing a monomer that has less acyl chloride groups during membrane fabrication, reduced surface carboxyl group density of polyamide TFC NF membranes can also be reached by innovative modifications to the polyamide selective layer that convert the carboxyl groups into other moieties.

\section{ASSOCIATED CONTENT}

\section{Supporting Information}

One table, detailing interfacial polymerization protocols of hand-cast TFC NF membranes. This material is available free of charge via the Internet at http://pubs.acs.org.

\section{AUTHOR INFORMATION}

\section{Corresponding Author}

*E-mail: xhuang@tsinghua.edu.cn (X.H.); menachem. elimelech@yale.edu (M.E.). Phone: +86 1062772342 (X.H.); +1 2034322789 (M.E.).

\section{Notes}

The authors declare no competing financial interest.

\section{ACKNOWLEDGMENTS}

This work was supported in part by the WaterCAMPWS, a Science and Technology Center of Advanced Materials for the Purification of Water with Systems under the National Science Foundation Grant CTS-0120978, and Tsinghua University Initiative Scientific Research Program (No. 20121087922). We also acknowledge a graduate fellowship (to Y.M.) made by China Scholarship Council.

\section{REFERENCES}

(1) Herzberg, M.; Kang, S.; Elimelech, M. Role of extracellular polymeric substances (EPS) in biofouling of reverse osmosis membranes. Environ. Sci. Technol. 2009, 43 (12), 4393-4398.

(2) Ang, W. S.; Lee, S. Y.; Elimelech, M. Chemical and physical aspects of cleaning of organic-fouled reverse osmosis membranes. $J$. Membr. Sci. 2006, 272 (1-2), 198-210.

(3) Van der Bruggen, B.; Mänttäri, M.; Nyström, M. Drawbacks of applying nanofiltration and how to avoid them: A review. Sep. Purif. Technol. 2008, 63 (2), 251-263.

(4) Speth, T. F.; Summers, R. S.; Gusses, A. M. Nanofiltration foulants from a treated surface water. Environ. Sci. Technol. 1998, 32 (22), 3612-3617.

(5) Kasher, R.; Contreras, A. E.; Steiner, Z.; Miao, J.; Li, Q. L. Studying the role of common membrane surface functionalities on adsorption and cleaning of organic foulants using QCM-D. Environ. Sci. Technol. 2011, 45 (15), 6309-6315.

(6) Rahimpour, A.; Jahanshahi, M.; Mortazavian, N.; Madaeni, S. S.; Mansourpanah, Y. Preparation and characterization of asymmetric polyethersulfone and thin-film composite polyamide nanofiltration membranes for water softening. Appl. Surf. Sci. 2010, 256 (6), 16571663.

(7) Chiang, Y. C.; Hsub, Y. Z.; Ruaan, R. C.; Chuang, C. J.; Tung, K. L. Nanofiltration membranes synthesized from hyperbranched polyethyleneimine. J. Membr. Sci. 2009, 326 (1), 19-26.

(8) Saha, N. K.; Joshi, S. V. Performance evaluation of thin film composite polyamide nanofiltration membrane with variation in monomer type. J. Membr. Sci. 2009, 342 (1-2), 60-69.

(9) Singh, P. S.; Aswal, V. K. Compacted nanoscale blocks to build skin layers of reverse osmosis and nanofiltration membranes: A revelation from small-angle neutron scattering. J. Phys. Chem. C 2007, 111 (44), 16219-16226.

(10) Yang, F. J.; Zhang, S. H.; Yang, D. L.; Jian, X. G. Preparation and characterization of polypiperazine amide/PPESK hollow fiber composite nanofiltration membrane. J. Membr. Sci. 2007, 301 (1-2), $85-92$.

(11) Ang, W. S.; Elimelech, M. Protein (BSA) fouling of reverse osmosis membranes: Implications for wastewater reclamation. J. Membr. Sci. 2007, 296 (1-2), 83-92. 
(12) Hong, S. K.; Elimelech, M. Chemical and physical aspects of natural organic matter (NOM) fouling of nanofiltration membranes. $J$. Membr. Sci. 1997, 132 (2), 159-181.

(13) Khulbe, K. C.; Feng, C.; Matsuura, T. The art of surface modification of synthetic polymeric membranes. J. Appl. Polym. Sci. 2010, 115 (2), 855-895.

(14) Van der Bruggen, B. Chemical modification of polyethersulfone nanofiltration membranes: a review. J. Appl. Polym. Sci. 2009, 114 (1), 630-642.

(15) Tang, C. Y.; Kwon, Y. N.; Leckie, J. O. Fouling of reverse osmosis and nanofiltration membranes by humic acid: Effects of solution composition and hydrodynamic conditions. J. Membr. Sci. 2007, 290 (1-2), 86-94.

(16) Tang, C. Y.; Leckie, J. O. Membrane independent limiting flux for RO and NF membranes fouled by humic acid. Environ. Sci. Technol. 2007, 41 (13), 4767-4773.

(17) Kim, S. L.; Paul Chen, J.; Ting, Y. P. Study on feed pretreatment for membrane filtration of secondary effluent. Sep. Purif. Technol. 2002, 29 (2), 171-179.

(18) Wang, X. M.; Waite, T. D. Role of gelling soluble and colloidal microbial products in membrane fouling. Environ. Sci. Technol. 2009, 43 (24), 9341-9347.

(19) Grant, G. T.; Morris, E. R.; Rees, D. A.; Smith, P. J. C.; Thom, D. Biological interactions between polysaccharides and divalent cations: egg-box model. FEBS Lett. 1973, 32 (1), 195-198.

(20) Mo, Y.; Xiao, K.; Shen, Y.; Huang, X. A new perspective on the effect of complexation between calcium and alginate on fouling during nanofiltration. Sep. Purif. Technol. 2011, 82, 121-127.

(21) Fonseca, A. C.; Summers, R. S.; Greenberg, A. R.; Hernandez, M. T. Extra-cellular polysaccharides, soluble microbial products, and natural organic matter impact on nanofiltration membranes flux decline. Environ. Sci. Technol. 2007, 41 (7), 2491-2497.

(22) Liu, M. H.; Yu, S. C.; Yong, Z.; Gao, C. J. Study on the thin-film composite nanofiltration membrane for the removal of sulfate from concentrated salt aqueous: Preparation and performance. J. Membr. Sci. 2008, 310 (1-2), 289-295.

(23) Verissimo, S.; Peinemann, K. V.; Bordado, J. Influence of the diamine structure on the nanofiltration performance, surface morphology and surface charge of the composite polyamide membranes. J. Membr. Sci. 2006, 279 (1-2), 266-275.

(24) Zhang, W.; He, G. H.; Gao, P.; Chen, G. H. Development and characterization of composite nanofiltration membranes and their application in concentration of antibiotics. Sep. Purif. Technol. 2003, 30 (1), 27-35.

(25) Oh, N. W.; Jegal, J.; Lee, K. H. Preparation and characterization of nanofiltration composite membranes using polyacrylonitrile (PAN). II. Preparation and characterization of polyamide composite membranes. J. Appl. Polym. Sci. 2001, 80 (14), 2729-2736.

(26) Yoon, K.; Hsiao, B. S.; Chu, B. High flux nanofiltration membranes based on interfacially polymerized polyamide barrier layer on polyacrylonitrile nanofibrous scaffolds. J. Membr. Sci. 2009, 326 (2), 484-492.

(27) Cadotte, J. E. Reverse osmosis membrane. U.S. Patent 4039440, 1975

(28) Cadotte, J. E. Reverse osmosis membrane. U.S. Patent 4259183, 1979

(29) Sundet, S. A. Microporous support layer with interfacially polymerized copolyamide thereon. U.S. Patent 4626468, 1986

(30) Yu, S.; Ma, M.; Liu, J.; Tao, J.; Liu, M.; Gao, C. Study on polyamide thin-film composite nanofiltration membrane by interfacial polymerization of polyvinylamine (PVAm) and isophthaloyl chloride (IPC). J. Membr. Sci. 2011, 379 (1-2), 164-173.

(31) Zhou, Y.; Yu, S. C.; Liu, M. H.; Chen, H. L.; Gao, C. J. Effect of mixed crosslinking agents on performance of thin-film-composite membranes. Desalination 2006, 192 (1-3), 182-189.

(32) Wei, J.; Zhang, S. H.; Wu, C. N.; Jian, X. G. Relationship between interfacial polymerization monomer structure and separation properties of PPESK based composite membranes. Acta Polym. Sin. 2006, 2, 298-302.
(33) Khare, V. P.; Greenberg, A. R.; Krantz, W. B. Investigation of the viscoelastic and transport properties of interfacially polymerized barrier layers using pendant drop mechanical analysis. J. Appl. Polym. Sci. 2004, 94 (2), 558-568.

(34) Dey, T. K.; Bindal, R. C.; Prabhakar, S.; Tewari, P. K. Development, characterization and performance evaluation of positively-charged thin film-composite nanofiltration membrane containing fixed quaternary ammonium moieties. Sep. Sci. Technol. 2011, 46 (6), 933-943.

(35) Son, S. H.; Jegal, J. Preparation and characterization of polyamide reverse-osmosis membranes with good chlorine tolerance. J. Appl. Polym. Sci. 2011, 120 (3), 1245-1252.

(36) Konagaya, S.; Tokai, M. Synthesis of ternary copolyamides from aromatic diamine ( $m$-phenylenediamine, diaminodiphenylsulfone), aromatic diamine with carboxyl or sulfonic group (3,5-diaminobenzoic acid, 2,4-diaminobenzenesulfonic acid), and iso- or terephthaloyl chloride. J. Appl. Polym. Sci. 2000, 76 (6), 913-920.

(37) Petersen, R. J. Composite reverse-osmosis and nanofiltration membranes. J. Membr. Sci. 1993, 83 (1), 81-150.

(38) Mansourpanah, Y.; Madaeni, S. S.; Rahimpour, A. Preparation and investigation of separation properties of polyethersulfone supported poly(piperazineamide) nanofiltration membrane using microwave-assisted polymerization. Sep. Purif. Technol. 2009, 69 (3), 234-242.

(39) Yip, N. Y.; Tiraferri, A.; Phillip, W. A.; Schiffman, J. D.; Elimelech, M. High performance thin-film composite forward osmosis membrane. Environ. Sci. Technol. 2010, 44 (10), 3812-3818.

(40) Tiraferri, A.; Yip, N. Y.; Phillip, W. A.; Schiffman, J. D.; Elimelech, M. Relating performance of thin-film composite forward osmosis membranes to support layer formation and structure. $J$. Membr. Sci. 2011, 367 (1-2), 340-352.

(41) Tiraferri, A.; Elimelech, M. Direct quantification of negatively charged functional groups on membrane surfaces. J. Membr. Sci. 2012, 389, 499-508.

(42) Elimelech, M.; Chen, W. H.; Waypa, J. J. Measuring the zeta (electrokinetic) potential of reverse-osmosis membranes by a streaming potential analyzer. Desalination 1994, 95 (3), 269-286.

(43) Childress, A. E.; Elimelech, M. Effect of solution chemistry on the surface charge of polymeric reverse osmosis and nanofiltration membranes. J. Membr. Sci. 1996, 119 (2), 253-268.

(44) Mi, B.; Elimelech, M. Organic fouling of forward osmosis membranes: Fouling reversibility and cleaning without chemical reagents. J. Membr. Sci. 2010, 348 (1-2), 337-345.

(45) Lee, S.; Elimelech, M. Relating organic fouling of reverse osmosis membranes to intermolecular adhesion forces. Environ. Sci. Technol. 2006, 40 (3), 980-987.

(46) Adout, A.; Kang, S.; Asatekin, A.; Mayes, A. M.; Elimelech, M. Ultrafiltration membranes incorporating amphiphilic comb copolymer additives prevent irreversible adhesion of bacteria. Environ. Sci. Technol. 2010, 44 (7), 2406-2411.

(47) Li, Q. L.; Elimelech, M. Organic fouling and chemical cleaning of nanofiltration membranes: Measurements and mechanisms. Environ. Sci. Technol. 2004, 38 (17), 4683-4693.

(48) Schaep, J.; Van der Bruggen, B.; Vandecasteele, C.; Wilms, D. Influence of ion size and charge in nanofiltration. Sep. Purif. Technol. 1998, 14 (1-3), 155-162.

(49) Yaroshchuk, A. E. Dielectric exclusion of ions from membranes. Adv. Colloid Interface Sci. 2000, 85 (2-3), 193-230.

(50) Bandini, S.; Vezzani, D. Nanofiltration modeling: the role of dielectric exclusion in membrane characterization. Chem. Eng. Sci. 2003, 58 (15), 3303-3326.

(51) Szymczyk, A.; Fievet, P. Investigating transport properties of nanofiltration membranes by means of a steric, electric and dielectric exclusion model. J. Membr. Sci. 2005, 252 (1-2), 77-88.

(52) Seidel, A.; Waypa, J. J.; Elimelech, M. Role of charge (Donnan) exclusion in removal of arsenic from water by a negatively charged porous nanofiltration membrane. Environ. Eng. Sci. 2001, 18 (2), 105113. 
(53) Mayes, A. M.; Asatekin, A.; Seoktae, K.; Elimelech, M. Antifouling ultrafiltration membranes containing polyacrylonitrile-graftpoly(ethylene oxide) comb copolymer additives. J. Membr. Sci. 2007, 298 (1-2), 136-146.

(54) Listiarini, K.; Chun, W.; Sun, D. D.; Leckie, J. O. Fouling mechanism and resistance analyses of systems containing sodium alginate, calcium, alum and their combination in dead-end fouling of nanofiltration membranes. J. Membr. Sci. 2009, 344 (1-2), 244-251. (55) Al-Amoudi, A.; Lovitt, R. W. Fouling strategies and the cleaning system of NF membranes and factors affecting cleaning efficiency. J. Membr. Sci. 2007, 4-28.

(56) Vrijenhoek, E. M.; Hong, S.; Elimelech, M. Influence of membrane surface properties on initial rate of colloidal fouling of reverse osmosis and nanofiltration membranes. J. Membr. Sci. 2001, 188 (1), 115-128. 